\title{
Rapid adiabatic passage in quantum dots: Influence of scattering and dephasing
}

\author{
Schuh, K.; Jahnke, F.; Lorke, Michael
}

Published in:

Applied Physics Letters

Link to article, DOI:

10.1063/1.3609016

Publication date:

2011

Document Version

Publisher's PDF, also known as Version of record

Link back to DTU Orbit

Citation (APA):

Schuh, K., Jahnke, F., \& Lorke, M. (2011). Rapid adiabatic passage in quantum dots: Influence of scattering and dephasing. Applied Physics Letters, 99(1), 011105. https://doi.org/10.1063/1.3609016

\section{General rights}

Copyright and moral rights for the publications made accessible in the public portal are retained by the authors and/or other copyright owners and it is a condition of accessing publications that users recognise and abide by the legal requirements associated with these rights.

- Users may download and print one copy of any publication from the public portal for the purpose of private study or research.

- You may not further distribute the material or use it for any profit-making activity or commercial gain

- You may freely distribute the URL identifying the publication in the public portal

If you believe that this document breaches copyright please contact us providing details, and we will remove access to the work immediately and investigate your claim. 


\title{
Rapid adiabatic passage in quantum dots: Influence of scattering and dephasing
}

\author{
K. Schuh, ${ }^{1, a)}$ F. Jahnke, ${ }^{1}$ and M. Lorke ${ }^{2}$ \\ ${ }^{1}$ Institute for Theoretical Physics, University of Bremen, P.O. Box 330440, 28334 Bremen, Germany \\ ${ }^{2}$ DTU Fotonik, Department of Photonics Engineering, Technical University of Denmark, Building 343 , \\ 2800 Kgs. Lyngby, Denmark
}

(Received 5 May 2011; accepted 16 June 2011; published online 6 July 2011)

\begin{abstract}
Theoretical investigations for the realization of population inversion of semiconductor quantum dot ground-state transitions by means of adiabatic passage with chirped optical pulses are presented. While the inversion due to Rabi oscillations depends sensitively on the resonance condition, the pulse area, as well as on the absence of carrier scattering and dephasing, we find that adiabatic passage is surprisingly insensitive to the excitation conditions and carrier scattering effects. Quantum kinetic models for the interaction of quantum-dot carriers with longitudinal optical phonons are used to describe carrier scattering and dephasing in the corresponding simulations and allow to quantify the conditions to simultaneously invert an ensamble of quantum dots. () 2011 American Institute of Physics. [doi:10.1063/1.3609016]
\end{abstract}

Photonic circuits, in which laser pulses are redirected by other laser pulses, are a long-standing goal for developing highly integrated optical communication components. A central part of these circuits is all-optical switches that have been demonstrated, e.g., using semiconductor optical amplifiers ${ }^{1,2}$ or photonic crystals. ${ }^{3}$ The latter approach has been based on semiconductor quantum dots (QDs), which are man-made semiconductor structures that possess a threedimensional carrier confinement.

In single QD, ultrafast optical switching can be achieved in the coherent regime with short laser pulses by utilizing to Rabi oscillations between the QD ground state of electrons and holes. A major problem is the omnipresence of dephasing in QD systems, which results in incomplete inversion. As QDs are embedded in a semiconductor matrix, the interaction with phonons leads to important dephasing contributions and the influence of both longitudinal acoustic (LA) and longitudinal optic (LO) phonons on Rabi oscillations in QDs has been investigated. ${ }^{4-8}$ Moreover, the interaction with LO phonons is also responsible for efficient carrier scattering ${ }^{9}$ that influences the inversion. A further hindrance for the use of Rabi oscillations in QD ensembles is the presence of inhomogeneous broadening as an unavoidable consequence of the growth process. As the dipole matrix elements of the QD transitions as well as the transition energies vary between different dots, a pulse that is tailored to provide full inversion for one QD will only lead to incomplete inversion in other QDs.

In the past, various schemes for ultrafast adiabatic population transfer have been studied in semiconductors, which are based, e.g., on stimulated Raman adiabatic passage ${ }^{10,11}$ or on the application of a chirped optical pulse that is swept through the resonance of an optical transition. ${ }^{12-14}$ In this paper, we focus on the latter scheme in order to invert the QD ground-state transition. In contrast to resonant $\pi$-pulses, which are applied for inversion via Rabi-flopping, adiabatic passage (AP) by means of a quasi-resonant chirped laser

${ }^{\text {a)} E l e c t r o n i c ~ m a i l: ~ k s c h u h @ i t p . u n i-b r e m e n . d e . ~}$ pulse is rather robust to pulse parameters like pulse area and frequency. The requirements of an adiabatic regime are shown for example in Ref. 15: A high squared peak Rabi frequency and a low squared inverse pulse length compared to the frequency-chirp rate are needed.

In this paper, we study the influence of carrier scattering on the efficiency of AP in QD systems. Carrier scattering leads to population redistribution and to dephasing of the induced optical polarization. Both effects are detrimental to a coherent state manipulation. We investigate AP for selfassambled InGaAs QDs with two confined shells for electrons as well as holes. The inhomogeneously broadened QD ensemble is randomly distributed on a wetting layer (WL). The excitation of carriers is modeled by the semiconductor Bloch equations ${ }^{16}$ including Coulomb renormalizations and quantum kinetic collision terms due to carrier-phonon interaction. In this paper we focus on the role of carrier scattering due to LO phonons. In contrast, LA phonons only contribute to pure dephasing and not to carrier scattering, since their QD interaction matrix elements favor small phonon momenta that correspond to phonon energies considerably smaller than the QD level separation. As long as there are efficient scattering channels for the LO phonons (typically at elevated temperatures), their influence on dephasing outweigh the contribution due to LA phonons. The used quantum kinetic treatment ${ }^{9}$ includes non-Markovian effects as well as non-perturbative energy renormalizations of the QD levels. Although, the level spacings of the QD states (60 meV for electrons and $25 \mathrm{meV}$ for holes) do not match the LO-phonon energy $(36 \mathrm{meV})$ there is efficient scattering between the QD states because of non-Markovian effects as well as quasiparticle renormalizations, which invalidate the free particle picture. ${ }^{9}$ Moreover, there is an efficient interaction with the nearby continuum of WL states that is explicitly included in our calculations.

While inversion can be achieved in QD systems with weak dephasing by an optical pulse with the length of several ps (Refs. 12 and 13), this is not possible for systems with efficient dephasing and scattering. Such systems require a 


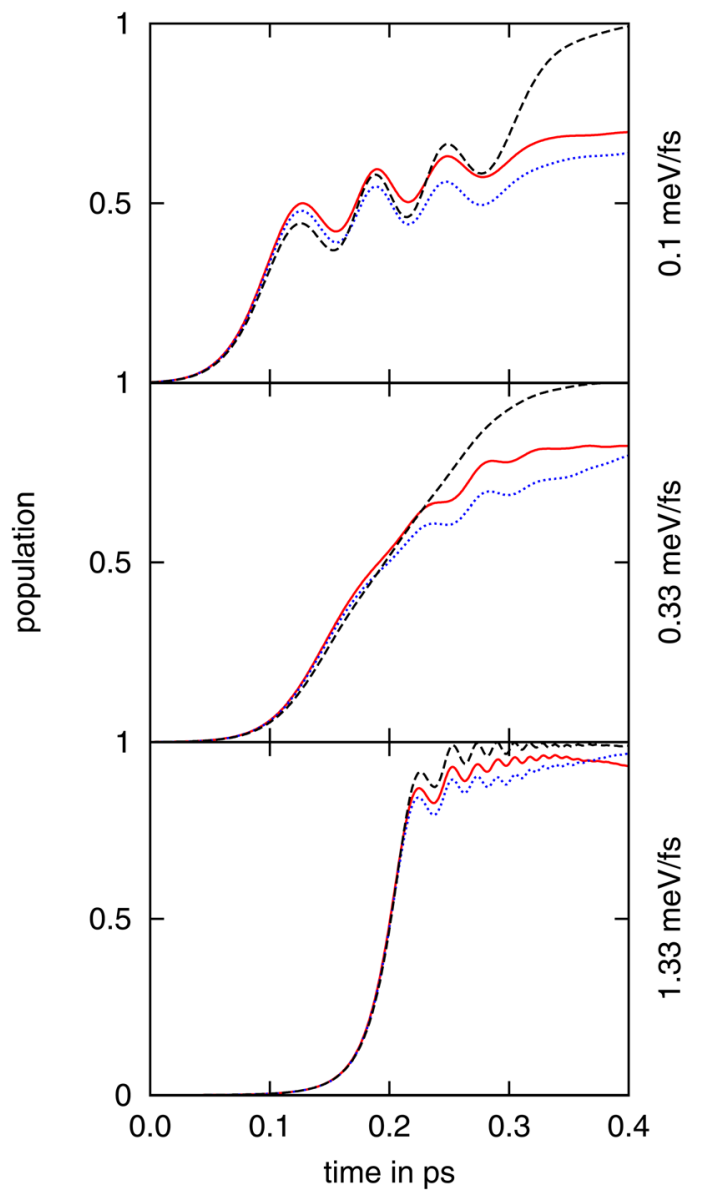

FIG. 1. (Color online) Temporal evolution of the population of the QD electron (solid line) and hole (dotted line) ground state being partially inverted by differently chirped $\left(0.1 \frac{\mathrm{meV}}{\mathrm{fs}}, 0.33 \frac{\mathrm{meV}}{\mathrm{fs}}\right.$, and $\left.1.33 \frac{\mathrm{meV}}{\mathrm{fs}}\right) 8 \pi$ pulses at a temperature of $77 \mathrm{~K}$. The results are compared with a two-level system (dashed line) for which energy renormalization is considered, but carrier scattering and dephasing due to LO phonons is neglected. The latter clearly reduces the inversion in the QD system for small chirp rates.

faster inversion in order to reduce the effect of dephasing during the inversion process. Thus, a strong chirp as well as a short pulse width are beneficial since they speed up the transition.

Figure 1 shows the temporal evolution of the groundstate carrier population for the investigated QD system due to differently chirped 200 fs pulses with a pulse area of $8 \pi$ that are illustrated in Fig. 2. For comparison, the dashed lines in Fig. 1 exhibit the upper-level population for a two-level system subject to the same energy renormalization but without carrier scattering and the resulting dephasing. The population oscillations during the transition of the two-level system reveal that only the dynamics at the intermediate chirp rate of $0.33 \frac{\mathrm{meV}}{\mathrm{fs}}$ is fully governed by the adiabatic regime. In the bottom picture, the faster chirp rate is too high compared to the squared peak Rabi frequency, and in the top picture the slower chirp is too small compared to the squared inverse pulse length in order to be in the adiabatic regime. Still, there is only a moderate reduction of the inversion for a wide area of chirp rates.

In contrast, the inversion of the investigated QD system keeps improving for further rising chirp rates due to a reduced influence of dephasing that is caused by the faster transition from ground state to inversion. Carrier scattering

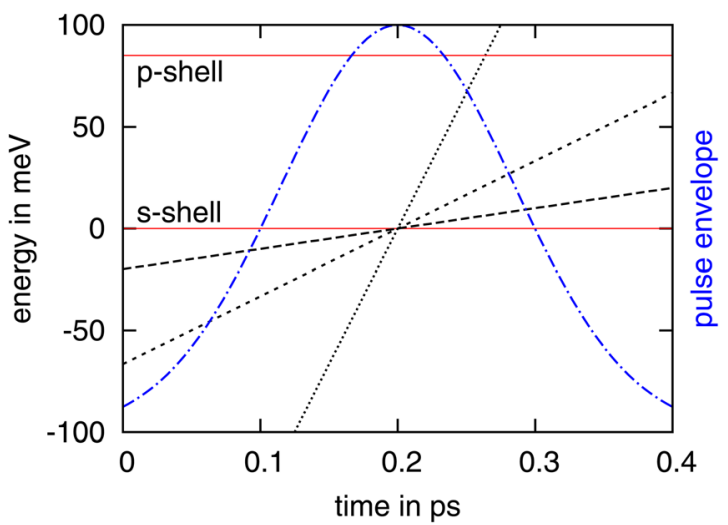

FIG. 2. (Color online) Excitation scheme with QDs and p-shell interband transitions (solid lines) and carrier frequency of three different chirped excitation pulse. At the temporal maximum of the pulse envelope (dashed dotted line) the pulses are in resonance with the s-shell transition. Different chirp rates correspond to Fig. 1 (long dashed $0.1 \frac{\mathrm{meV}}{\mathrm{fs}}$, short dashed $0.33 \frac{\mathrm{meV}}{\mathrm{fs}}$, dotted $\left.1.33 \frac{\mathrm{meV}}{\mathrm{fs}}\right)$. For the largest chirp rate, also the p-transition is excited.

is present even after the optical transition is completed, but its effect on the resulting inversion is much smaller than that of dephasing for the investigated QDs. Thus, to achieve a high inversion a strong chirp is beneficial that reduces dephasing but is not so high that the violation of the adiabatic regime compromises the effect. An example for this is shown in Fig. 1 for a chirp rate of $1.33 \frac{\mathrm{meV}}{\mathrm{fs}}$.

Different level spacings for electrons and holes are the main origin for deviations in the corresponding scattering rates, thus leading to independently evolving electron and hole populations. For instance, during the pulse the out-scattering of holes is faster because of the smaller energy difference of the hole ground state to the exited state as well as to the WL states compared to the electrons. Then during the pulse the hole population remains smaller than the electron population. Since in this case the smaller energy difference causes also faster in-scattering and the strongly chirped pulses also invert the higher QD states, the hole population can overtake the electron population after the pulse due to the redistribution of carriers from excited states. In this respect our results deviate from the inversion of a certain single transition like Melinger et al. ${ }^{14}$ demonstrated for molecules with a longer pulse.

Figure 3 shows the stability of the inversion of the QD ground state for a large interval of pulse peak frequencies. Using a linearly chirped pulse, this interval has about the size of two times the chirp (chirp rate times pulse length). Thus, for the two-level system there is nearly a full inversion in this interval. For the QD system, the inversion of the ground state is reduced in a similar fashion as in Figure 1. Moreover, in this case the symmetry of the curve is absent due to scattering with energetically higher states that become also excited by the high energy components of the chirped pulse. Depending on the excitation of other states, carrier scattering can increase or decrease the resulting inversion because a higher population of the other states increases inscattering and decreases out-scattering from the investigated ground state.

An important property of the discussed population inversion of QDs due to AP is its weak dependency on the 


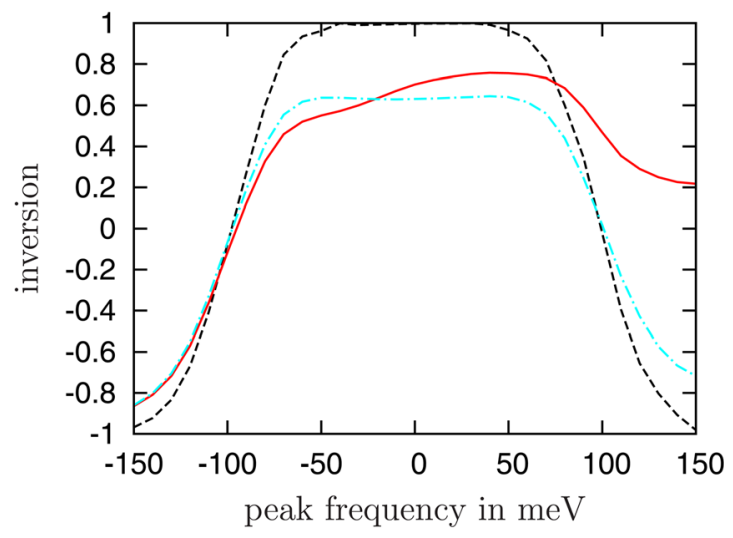

FIG. 3. (Color online) Resulting inversion versus pulse frequency at peak intensity for a two-level system (dashed line) and QD ground state (solid line) (pulse area $=6 \pi$, chirprate $=0.33 \frac{\mathrm{meV}}{\mathrm{fs}}$ ). The dashed-dotted line shows the QD ground state without carrier scattering but with dephasing due to carrier-LO-phonon interaction. A high degree of inversion is possible with a single pulse for QDs with very different transition energies.

pulse area shown in Figure 4. For resonant unchirped excitation, we observe Rabi oscillations that do not lead to full inversion due to dephasing. Moreover, as a result of manybody renormalizations, the extrema of the inversion do not occur at multiples of $\pi$. For chirped excitation the inversion depends only weakly on the pulse area, provided that a certain minimal pulse area is exceeded. This is caused by the conditions of the adiabatic regime and leads to the fact that the required minimum pulse area increases approximately quadratically with increasing chirp rate. For large pulse areas increased chirp rates lead to higher values of the inversion, as the transition is less influenced by dephasing. In contrast, for small pulse areas the resulting inversion is higher for smaller chirp rates, as in this case the violation of the adiabatic regime is not so severe.

In conclusion, AP is a useful tool for the generation of population inversion in a QD ensemble due to the stability against detuning of the QD transitions and variations of the dipole coupling of the individual QDs. Moreover, our calculations show that even in QD systems with fast dephasing and carrier scattering due to carrier interaction with LO phonons, high inversion can be achieved by AP. However, this requires a fast transition that can be achieved by a large frequency chirp, even though this violates to some extent the adiabatic regime. While the dephasing is the dominant source for the reduction of the resulting inversion, the contribution due to carrier scattering can increase or decrease the inversion, depending on the population of the other QD states, which are also excited by the chirped pulse.

This work was supported by the Deutsche Forschungsgemeinschaft and the Danish Research Council for Technol-

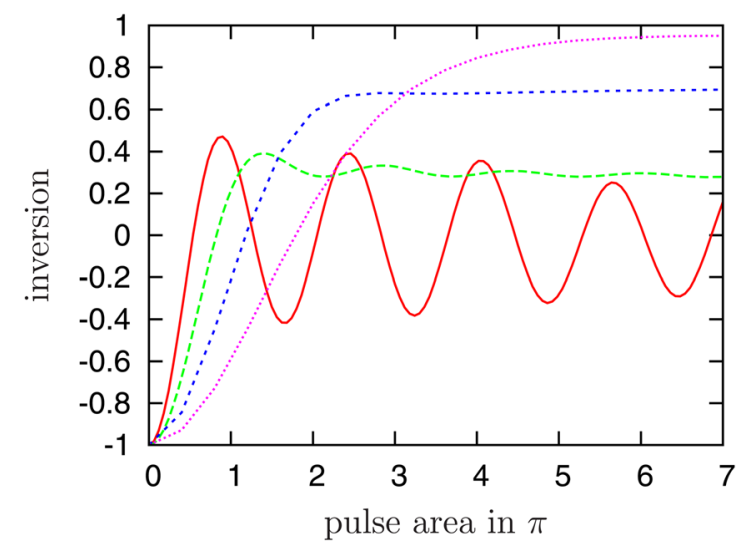

FIG. 4. (Color online) Inversion of the QD ground state as a result of a resonant (solid line) or chirped (dotted and dashed lines) 200fs pulse versus its unrenormalized pulse area: While there is an oscillatory dependency for the resonant pulse, the inversion caused by a chirped pulse depends only slightly on its pulse area. With increasing chirp rate (long dashed $0.1 \frac{\mathrm{meV}}{\mathrm{fs}}$, short dashed $0.33 \frac{\mathrm{meV}}{\mathrm{fs}}$, dotted $1.33 \frac{\mathrm{meV}}{\mathrm{fs}}$ ) the influence of the dephasing is reduced and therefore the inversion is increased.

ogy and Production under Project No. 09-070892. M. Lorke is grateful to the "Young Elite Researcher Award" initiative by the Danish Council for Independent Research.

${ }^{1}$ H. Ju, S. Zhang, D. Lenstra, H. de Waardt, E. Tangdiongga, G. Khoe, and H. Dorren, Opt. Express 13, 942 (2005).

${ }^{2}$ M. L. Nielsen, J. Mørk, R. Suzuki, J. Sakaguchi, and Y. Ueno, Opt. Express 14, 331 (2006).

${ }^{3}$ R. Prasanth, J. E. M. Haverkort, A. Deepthy, E. W. Bogaart, J. J. G. M. van der Tol, E. A. Patent, G. Zhao, Q. Gong, P. J. van Veldhoven, R. Nötzel, and J. H. Wolter, Appl. Phys. Lett. 84, 4059 (2004).

${ }^{4}$ J. Förstner, C. Weber, J. Danckwerts, and A. Knorr, Phys. Stat. Sol. B 238 , 419 (2003).

${ }^{5}$ Q. Q. Wang, A. Muller, P. Bianucci, E. Rossi, Q. K. Xue, T. Takagahara, C. Piermarocchi, A. H. MacDonald, and C. K. Shih, Phys. Rev. B 72, 035306 (2005).

${ }^{6}$ A. Vagov, M. Croitoru, V. Axt, and T. K. F. Peeters, Phys. Stat. Sol. B 243, 2233 (2006).

${ }^{7}$ A. J. Ramsay, A. V. Gopal, E. M. Gauger, A. Nazir, B. W. Lovett, A. M. Fox, and M. S. Skolnick, Phys. Rev. Lett. 104, 017402 (2010).

${ }^{8}$ K. Schuh, J. Seebeck, M. Lorke, and F. Jahnke, Appl. Phys. Lett. 94, 201108 (2009).

${ }^{9}$ J. Seebeck, T. Nielsen, P. Gartner, and F. Jahnke, Phys. Rev. B 71, 125327 (2005).

${ }^{10}$ U. Hohenester, J. Fabian, and F. Troiani, Opt. Commun. 264, 426 (2006).

${ }^{11}$ R. Binder and M. Lindberg, Phys. Rev. Lett. 81, 1477 (1998).

${ }^{12}$ C.-M. Simon, T. Belhadj, B. Chatel, T. Amand, P. Renucci, A. Lemaitre, O. Krebs, P. A. Dalgarno, R. J. Warburton, X. Marie, and B. Urbaszek, e-print arXivp.1007.2808v1.

${ }^{13}$ E. R. Schmidgall, P. R. Eastham, and R. T. Phillips, Phys. Rev. B 81, 195306 (2010).

${ }^{14}$ J. S. Melinger, S. R. Gandhi, A. Hariharan, J. X. Tull, and W. S. Warren, Phys. Rev. Lett. 68, 2000 (1992).

${ }^{15}$ V. S. Malinovsky and J. L. Krause, Eur. Phys. J. D 14, 147 (2001).

${ }^{16} \mathrm{H}$. Haug and S. Koch, Quantum Theory of the Optical and Electronic Properties of Semiconductors, 4th ed. (World Scientific Publishing, Singapore, 2004). 